\title{
Examination of Mycobacterium tuberculosis sigma factor mutants using low-dose aerosol infection of guinea pigs suggests a role for SigC in pathogenesis
}

\author{
Russell K. Karls, ${ }^{1,2}$ Jeannette Guarner, ${ }^{3}$ David N. McMurray, ${ }^{4}$ \\ Kristin A. Birkness ${ }^{2}$ and Frederick D. Quinn ${ }^{1,2}$ \\ ${ }^{1}$ Department of Infectious Diseases, College of Veterinary Medicine, University of Georgia, \\ Athens, GA 30602, USA \\ ${ }^{2}$ Mycobacteriology Laboratory Branch, Division of Tuberculosis Elimination, National Center for \\ HIV, STD, and TB Prevention, Centers for Disease Control and Prevention, Atlanta, GA \\ 30333, USA \\ ${ }^{3}$ Infectious Disease Pathology Activity, National Center for Infectious Diseases, Centers for \\ Disease Control and Prevention, Atlanta, GA 30333, USA \\ ${ }^{4}$ Department of Medical Microbiology and Immunology, Texas A\&M University System Health \\ Science Center, College Station, TX 77845, USA
}

Correspondence

Frederick D. Quinn

fquinn@vet.uga.edu

Received 14 October 2005

Revised 16 February 2006

Accepted 22 February 2006

\section{INTRODUCTION}

Tuberculosis (TB) remains a major health problem in most parts of the world. Despite decades of effort to eliminate the disease, Mycobacterium tuberculosis infection remains the leading cause of death and disease by a single bacterial human pathogen, claiming approximately two million lives each year (World Health Organization, 2005; Dye et al., 1999). Although the M. bovis BCG vaccine is widely used to prevent mycobacterial meningitis in children, its efficacy against pulmonary TB is highly variable $(0-80 \%$; World Health Organization, 1979; Rodrigues \& Smith, 1990).

Abbreviations: AFB, acid-fast bacilli; TB, tuberculosis.
Understanding how $M$. tuberculosis bacilli adapt and survive upon encountering hostile environments inside the host is critical to producing effective vaccines and therapies. Bacteria employ multiple secondary sigma factors that sense specific stress signals and transiently coordinate expression of genes encoding functions that facilitate bacterial adaptation to those particular stresses. Sequence analysis of the M. tuberculosis strain H37Rv suggested the presence of 12 secondary sigma factor genes $(\operatorname{sig} B-s i g M)$ and a primary sigma factor gene, sigA (Cole et al., 1998).

Studies of knockout mutations of secondary sigma factor genes in M. tuberculosis strains suggest some sigma factors play roles in virulence. While the $s i g D$ and sigF genes are apparently not required for survival in macrophage infections, a sigD mutant of H37Rv and a sigF mutant of 
strain CDC1551 were each attenuated for pathogenesis in BALB/c mice (Raman et al., 2004; Chen et al., 2000). The sigF mutant was subsequently shown to exhibit reduced persistence in the lungs and spleen of BALB/c mice (Geiman et al., 2004). A sigH mutant of CDC1551 survived in macrophages and persisted in the lungs and spleen of C57BL/6 mice, suggesting that SigH had no role in vivo. However, in $\mathrm{C} 3 \mathrm{H} / \mathrm{HeJ}$ mice, $\mathrm{SigH}$ contributed to tissue damage and death of the host (Kaushal et al., 2002). A sigC mutant of CDC1551 was reported to be less virulent, but just as persistent as the parental strain in DBA/2 mice (Sun et al., 2004). SigJ appeared not to be required for survival in BALB/ c mice (Hu et al., 2004). SigE, however, was reported to be required for survival of $\mathrm{H} 37 \mathrm{Rv}$ in macrophages (Manganelli et al., 2001). In addition, a sigE mutant persisted less effectively than the $\mathrm{H} 37 \mathrm{Rv}$ parent strain in the lungs, spleen and liver of BALB/c mice, and mice infected with the mutant survived longer than those infected with the parent strain (Manganelli et al., 2004). Interestingly, while $\mathrm{C} 3 \mathrm{H} / \mathrm{HeJ}$ mice infected with a sigE mutant of the CDC1551 strain also survived longer than those infected with the parent strain, the mutant was not impaired for lung persistence (Ando et al., 2003). Genetic differences between the parent strains of the sigE mutants or differential susceptibilities in the murine models were posited to explain the observed disparity in persistence (Manganelli et al., 2004). Such phenotypic variability highlights the importance of examining mutants in different genetic backgrounds and the need for study in multiple animal model systems.

While murine models of TB have the advantages of low cost, availability of immunological reagents, and the choice of inbred populations with varying susceptibility to aerosol infection, guinea pigs are considered a superior model of human M. tuberculosis infection in terms of lung granuloma formation and function, subsequent necrosis, caseation and disease progression (Baldwin et al., 1998; Turner et al., 2003; McMurray et al., 1996; Orme et al., 2001; Ridley \& Ridley, 1987). The classical human lung granuloma contains a caseous necrotic core with varying degrees of calcification surrounded by epithelioid macrophages and layers of T lymphocytes and fibrous proteins (Cotran et al., 1999). While these features are evident in the guinea pig TB model, the same does not hold for murine models. Murine granulomas are structured differently, containing significant numbers of B lymphocytes (Gonzalez-Juarrero et al., 2001). Mice also produce an unusually strong cellular immune response to $M$. tuberculosis bacilli that limits lung damage and enables sustained bacillary loads in excess of $10^{6}$ per lung for many weeks (Orme et al., 2001). In addition, it may take months, if ever, for murine granulomas to exhibit central necrosis, but calcification never occurs (Rhoades et al., 1997).

In this study, we employed low-dose aerosol infection of guinea pigs for screening sigma factor knockout mutants of strain H37Rv to identify sigma factors that may be important regulators of virulence. Mutants defective in production of SigC produced the most-attenuated phenotypes, exhibiting limited pathogenesis in the lungs and spleen. This work suggests that $\mathrm{SigC}$ has a role in granuloma formation in the lungs and in successful dissemination and establishment of infection in other organs.

\section{METHODS}

Enzymes and reagents. High-fidelity $P f u$ DNA polymerase was purchased from Stratagene. Restriction endonucleases, DNAmodifying enzymes, nucleotides and DNA standards were purchased from New England Biolabs. Antibiotics were purchased from Research Products International. Primers were synthesized by the Centers for Disease Control and Prevention (CDC) core facility or purchased from Integrated DNA Technologies. Primers used in these studies are indicated in Table 1.

Bacterial strains. All M. tuberculosis mutant strains in this report were derived from virulent strain $\mathrm{H} 37 \mathrm{Rv}$ obtained from the CDC mycobacterial strain collection. Mycobacterial strains were cultured in Dubos medium supplemented with OADC (BD/Difco) and 0.05\% Tween 80 . Replicate bacterial stocks used for infections were prepared from cultures grown to exponential phase $\left(\mathrm{OD}_{580} 0 \cdot 5\right.$, measured with a Thermo-Spectronic 20D + spectrophotometer). Bacterial titres were obtained from thawed stocks after serial dilution in culture medium and plating on Middlebrook 7H11 agar with OADC supplementation (BD/Difco). When appropriate, the antibiotic kanamycin or hygromycin was used at 25 and $50 \mu \mathrm{g} \mathrm{ml}^{-1}$, respectively.

Plasmid constructions. Plasmid pPR27 $\Delta$ sigMhyg, used to create a M. tuberculosis sigM deletion mutant (sigMhyg) marked with a hygromycin resistance gene from p16R1, hyg, was made as follows. The sigM gene $+\sim 500$ bp flanking regions obtained by PCR with M. tuberculosis DNA template, Pfu DNA polymerase, dNTPs and primers sigMF1/sigMR1 was cloned into plasmid pCR2.1TOPO (Invitrogen). An internal deletion of sigM was obtained by digesting the resulting plasmid with SacII and AgeI, blunting the DNA ends with T4 DNA polymerase, and ligating with the blunted $\sim 1300$ bp BspHI-SmaI hyg gene from p16R1 (Yuan et al., 1998) to form

Table 1. Primers

\begin{tabular}{|ll|}
\hline Primer & \multicolumn{1}{c|}{ Sequence $\left(\mathbf{5}^{\prime} \mathbf{- 3}^{\prime}\right)$} \\
\hline sigCF1 & GGGATCCTGAACGTGTTGTTGGGCCA \\
sigCR 1 & GGGATCCGGTACCGCCA \\
sigMF1 & GGACTAGTCTTGGCTTCGGTGTTGA \\
sigMR 1 & GGACTAGTGTGGTGAAGCAGGTCGA \\
usfXF1 & ATGGATCCGGTGCTGACGCTCTGCATAG \\
sigFR5 & GAGGATCCTCGGTACATACCCGCTGG \\
sigF $\Delta$ F1 & CCTTAATTAACGTCGGCGTATTCGTTAGCT \\
sigF $\Delta$ R1 & CCTTAATTAAGTCATTGGCACGGCTACGG \\
up565sigC & GGGGTACCGTCGCTATAGCGTATC \\
down92sigC & CGCAAGCTTCGGTGGTCATGATAGC \\
sigMoutF1 & GAGTCAAGCCGACATCTCG \\
sigMoutR1 & GATCGATGCTACGACGGAC \\
sigFoutF1 & CATCGCGAGCTAACGAATAC \\
sigFoutR1 & CGTAGCCGTGCCAATGACT \\
sigCoutF1 & CACCCAAGAGACATTCCTAC \\
sigCoutR1 & TTACCACGATGAGTTCGCAC \\
sigC10F & ACGGCAAGCGACGACGAG \\
sigC379R & TCGTGGTTACCTCGACGAG \\
\hline
\end{tabular}


plasmid pCR2.1 $\Delta$ sigMhyg. The SpeI fragment containing the $\Delta$ sigMhyg $+\sim 500$ bp flanking regions was then cloned into plasmid pPR27 (Pelicic et al., 1997) to form plasmid pPR27 $\Delta$ sigMhyg.

Plasmid pPR27 $\Delta$ sigFhyg, used in the creation of a hyg-marked sigF deletion mutant in $M$. tuberculosis (sigFhyg), was produced as follows. The $s i g F$ gene plus flanking regions (1140 bp upstream, 500 bp downstream), obtained by PCR from M. tuberculosis template with primers usfXF1/sigFR5, was TA-cloned into plasmid pCR2.1TOPO, creating plasmid pRKK413. The sigF gene was deleted from this plasmid by PCR using primers $\operatorname{sigF} \Delta \mathrm{F} 1 / \operatorname{sigF} \Delta \mathrm{R} 1$ to form plasmid pCR2.1 $\Delta$ sigF1. The hyg gene was inserted into the PacI site of pRKK413 to create pCR2.1 $\Delta$ sigFhyg. The BamHI fragment containing $\Delta$ sigFhyg plus flanking sequences was then ligated into the BamHI site of pPR27 to form pPR27 $\Delta$ sigFhyg.

Plasmid pPR27sigChyg, used in generating a hyg-marked disruption mutation in the SigC-coding region of M. tuberculosis (sigChyg), was constructed as follows. A DNA segment carrying sigC plus $\sim 500$ bp flanking regions obtained from $M$. tuberculosis template DNA (by PCR with primers sigCF1 and sigCR1) was TA-cloned into vector pCR2.1TOPO to form plasmid pCR2.1sigC. A 1293 bp hygromycin resistance gene, $h y g$, obtained as a $S m a \mathrm{I}-B s p \mathrm{HI}$ fragment from plasmid p16R1, was inserted into the unique EcoNI site within sigC to form plasmid pCR2.1sigChyg. The BamHI fragment encoding the hyg-disrupted sigC gene plus $\sim 500$ bp flanking regions from this plasmid was ligated into plasmid pPR27 to form pPR27sigChyg.

Plasmid $\mathrm{pLJ} \Delta$ sigC used in the creation of an unmarked internal sigC deletion mutant of $M$. tuberculosis $(R v$-sigC) was constructed as follows. The 1569 bp BamHI fragment containing sigC plus $\sim 500$ bp flanking regions was excised from pCR2.1sigC and inserted into the BamHI site of plasmid pUC19spf' (Erickson \& Gross, 1989). An internal deletion of the carboxy-terminal third of $\operatorname{sig} C$ was obtained by removing the sequence between the EcoNI and XhoI sites to form pUC19spf $\Delta$ sigC. The BamHI fragment containing $\Delta$ sigC plus $500 \mathrm{bp}$ flanking sequences was excised from this plasmid and ligated into the BamHI site of plasmid pLCG2 to form plasmid pLCG2 $\Delta$ sigC. Plasmid pLCG2 is a derivative of p2NIL (Parish \& Stoker, 2000) in which the PacI site was destroyed by insertion of a PmeI linker (New England Biolabs). The PacI fragment encoding the lac-sac region from pGOAL17 was then inserted into the PmeI site of pLCG2 $\Delta$ sigC to create $\mathrm{pLJ} \Delta \operatorname{sigC}$.

Complementation plasmid pMV306sigC was created as follows. The M. tuberculosis sigC region (containing 565 bp of upstream regulatory sequence to $92 \mathrm{bp}$ downstream of sigC), obtained by PCR using primers up565sigC/down92sigC, was ligated into the HindIII site of the mycobacterial integrating vector pMV306 (Kong \& Kunimoto, 1995).

Isolation of M. tuberculosis mutants. The hygromycin-marked M. tuberculosis sigma factor knockout strains (sigChyg, sigFhyg, sigMhyg) were obtained using the homologous recombination method of Pelicic et al. (1997) following electroporation of H37Rv with a plasmid derived from the mycobacterial temperature-sensitive replicating plasmid pPR27 (pPR27sigChyg, pPR27 $\Delta$ sigFhyg, pPR27$\Delta$ sigMhyg, respectively). The unmarked sigC mutant $(R v$-sigC) was obtained following electroporation of H37Rv with the suicide plasmid $\mathrm{pLJ} \Delta$ sigC using the unmarked deletion method (Parish \& Stoker, 2000). Strain sigChyg was complemented with a wild-type copy of the sigC gene by introduction of plasmid pMV306sigC, resulting in strain sigCcomp. All plasmids were sequenced to check for the absence of PCR-generated mutations.

THP-1 infections. The human monocytic cell line THP-1 was cultured in RPMI medium supplemented with $10 \%$ fetal bovine serum (RPMI $+10 \%$ FBS). For infection assays, $10^{6}$ monocytes were seeded into each well of 24 -well dishes. Differentiation into macrophages was achieved by incubation for 3 days following addition of $100 \mathrm{nM}$ phorbol esters (Sigma-Aldrich). The macrophage monolayers were incubated for $24 \mathrm{~h}$ in medium lacking phorbol esters prior to infection. Cells were infected for $6 \mathrm{~h}$ with the indicated $M$. tuberculosis strains at an m.o.i. of approximately 10 bacilli per host cell by addition of identical volumes from bacterial stocks previously stocked at identical cell densities $\left(\mathrm{OD}_{580}=0 \cdot 5\right)$. Nonadherent bacilli were removed by washing the monolayers with Hank's Balanced Salts Solution (HBSS, Invitrogen). External bacilli not removed by washing were prevented from replicating by $2 \mathrm{~h}$ incubation of the monolayers in RPMI $+10 \%$ FBS containing $200 \mu \mathrm{g}$ amikacin $\mathrm{ml}^{-1}$. The monolayers were again washed with HBSS and then covered with $1 \mathrm{ml}$ RPMI $+10 \%$ FBS. At the indicated timepoints, THP-1 cells were lysed by the addition of Triton $\mathrm{X}-100$ to $0 \cdot 1 \%$. Viable bacilli were enumerated by serial dilution in Middlebrook 7H9 medium containing $0.05 \%$ Tween 80 and supplemented with $10 \%$ ADC (BD/Difco) and plating on Middlebrook $7 \mathrm{H} 11$ agar supplemented with $10 \%$ OADC (BD/Difco) and $0.05 \%$ Tween 80 . Over the 7 day infection period, uninfected THP-1 monolayers progressed from $50 \%$ to almost complete confluence. Over a comparable period, infected monolayers remained at approximately $50 \%$ confluence although an increased number of detached cells were detected as the infections proceeded.

Animal infections. Female outbred Hartley guinea pigs ( $\sim 500 \mathrm{~g})$ used in this study were purchased from the Charles River Laboratories. M. tuberculosis $\mathrm{H} 37 \mathrm{Rv}$ and mutant derivatives were grown to exponential phase in Dubos medium containing 0.05\% Tween 80 and supplemented with $10 \%$ Middlebrook OADC (BD/Difco). Cultures were divided into $1 \mathrm{ml}$ aliquots and frozen at $-80{ }^{\circ} \mathrm{C}$ until used. Thawed aliquots were diluted in double-distilled sterile water to the desired inoculum concentration. Animals were infected using a Madison aerosol generation chamber (Wiegeshaus et al., 1970) calibrated to deliver approximately 30 c.f.u. M. tuberculosis bacilli into the lungs of each guinea pig. Except as otherwise indicated, four animals infected with each strain were sacrificed at each timepoint. The lower left lung lobe and distal third of the spleen from each animal were fixed in $10 \%$ formalin for $20 \mathrm{~h}$ and then placed in $70 \%$ ethanol. Tissues with the most pathology for each specimen were paraffin embedded and $4 \mu \mathrm{m}$ tissue sections were stained with haematoxylin and eosin for histopathology and Ziehl-Neelsen stains for acid-fast bacilli (AFB)

\section{RESULTS}

\section{Creation of sigma factor mutants}

We initially examined three sigma factor null strains: sigChyg, sigFhyg and sigMhyg. The mutant strains were created by insertion of a hygromycin resistance gene ( $h y g)$ within the coding region of the specified sigma factor gene ( $\operatorname{sig} C, \operatorname{sig} F$ and $s i g M$, respectively) in $M$. tuberculosis strain H37Rv. The orientation of the hyg gene relative to the sigma factor coding sequence and to adjacent genes on the chromosome is shown in Fig. 1. Mutants were identified by PCR and confirmed by Southern analysis (data not shown).

\section{Examination of in vitro growth characteristics and intracellular survival of sigma factor mutants}

General growth characteristics of the hygromycin-marked sigma factor mutant strains (sigChyg, sigFhyg and sigMhyg) and the H37Rv parent strain were monitored. 


\section{hyg}

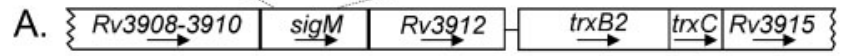

\section{hyg}

B.

\begin{tabular}{|l|l|l|l|}
\hline$R$ Rv3291c, lat, $R \nvdash 3289 c$, usfY & rbsWW & $\underline{\text { sigF }}$ & accA3 \\
\hline
\end{tabular}

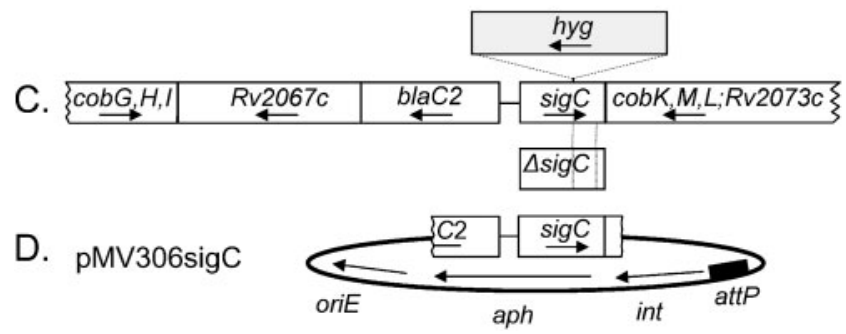

Fig. 1. (A, B, C) Sigma factor mutations and $P C R$ analysis. Sigma factor genes sigM, sigF and $\operatorname{sig} C$ are drawn with putative adjacent chromosomal ORFs based on information from the Tuberculist database. ORFs (boxes) are approximately to scale relative to each other. ORFs or multiple ORFs extending beyond the figure are indicated (jagged-ended boxes). The orientation of each ORF is indicated (arrows). Insertion sites of the hygromycin resistance gene, hyg, present in each of the marked sigma factor mutants (sigMhyg, sigFhyg, sigChyg) are indicated above each sigma factor gene (dashed lines). The internal region of sigC deleted in the unmarked $\operatorname{sig} C$ mutant strain $R v$-sigC is indicated by the shaded region within the $\Delta$ sigC box located below the sigC gene (C). (D) The sigC gene and flanking regions (boxes) cloned into the integrating plasmid pMV306 (thick circular line) used for complementation studies are indicated.

Mid-exponential-phase cultures $\left(\mathrm{OD}_{580}=0 \cdot 5\right)$ were used to inoculate $250 \mathrm{ml}$ capped side-arm flasks containing $50 \mathrm{ml}$ Middlebrook 7H9 plus ADC enrichment to an $\mathrm{OD}_{580}$ of 0.01 unit. Similar growth kinetics was observed among all of these shaking cultures, indicating that the mutants were not impaired for growth under these conditions (data not shown). Because survival in human macrophages is essential for M. tuberculosis disease progression, the mutants were next examined for the ability to replicate inside the human monocytic macrophage cell line THP-1 (Fig. 2). Initial differences at the $t=0$ timepoint may reflect differences in either self-adherence or adherence of the different strains to host cells following introduction into the cell culture medium as it lacks the anti-clumping non-ionic detergent Tween 80. However, each of the sigma factor knockout strains achieved similar titres by day 6 post-infection, suggesting that none are impaired in intracellular replication.

\section{Examination of guinea pig lung infection and dissemination to the spleen}

To determine which of the sigma factor mutants is required for adaptive survival upon entering a mammalian host,

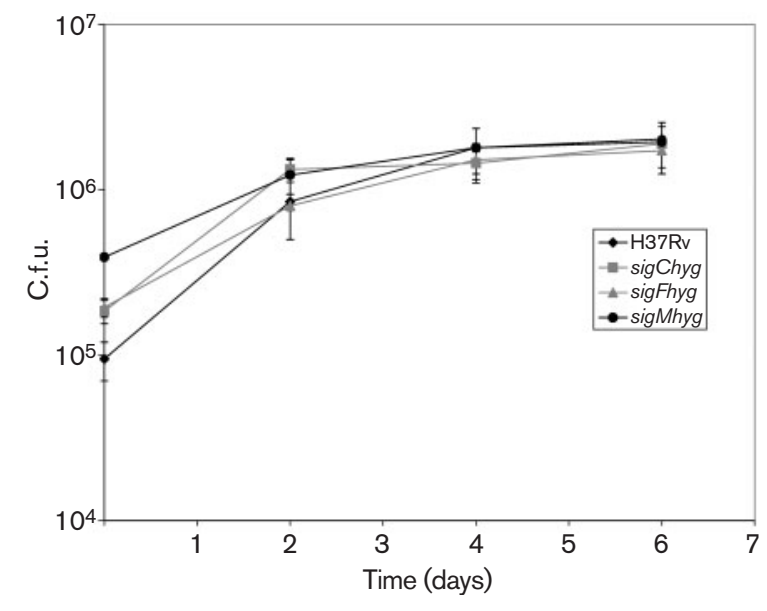

Fig. 2. Replication of $M$. tuberculosis strains in THP-1 macrophages. THP-1 macrophages were infected with the indicated strains at an m.o.i. of 10 for $6 \mathrm{~h}$, followed by $2 \mathrm{~h}$ incubation with amikacin $\left(200 \mathrm{~g} \mathrm{~m} \mathrm{~m}^{-1}\right)$ to prevent replication of external bacilli. After washing with HBSS, fresh medium was placed on the monolayers. Bacteria were enumerated at the indicated timepoints following lysis of monolayers with $0.1 \%$ Triton X100 , serial dilution in medium containing $0.05 \%$ Tween 80 and plating on Middlebrook $7 \mathrm{H} 11$ medium. Data presented are the mean of two experiments performed in triplicate. Error bars indicate standard deviation.

each was introduced into guinea pigs via the respiratory route using a Madison Chamber calibrated to deliver approximately 30 c.f.u. to the lungs. The infecting bacilli were previously grown to early exponential phase growth $\left(\mathrm{OD}_{580}=0 \cdot 5\right)$ in rich medium to minimize expression of individual stress-response sigma factor genes prior to infection. Animals were monitored over a 20 week period. The three marked sigma factor mutant strains sigMhyg, sigFhyg and sigChyg were initially compared against the parental strain H37Rv. Subsequently, two additional sigC mutant strains were tested. Strain sigCcomp complemented the marked sigChyg strain with a wild-type copy of sigC integrated at the mycobacteriophage L5 attachment site on the chromosome. Strain $R v$-sigC contains an unmarked internal deletion within sigC to control for the possibility that the sigChyg phenotypes were not caused by increased expression of blaC due to read-through from the hyg gene (see Fig. 1).

Histopathological analyses of the lungs and spleen were performed. In general for each strain, the granulomas in the lung and spleen at early timepoints (5-6 weeks postinfection) appeared to be larger (some having necrotic centres), while fewer granulomas with reduced pathology were observed at 20 weeks post-infection. The sigC mutants produced the least pathology of all of the strains examined for granuloma formation, necrosis and mineralization (Figs 3, 4 and 5, Table 2). While medium and large granulomas were detected in the lungs of animals at both 

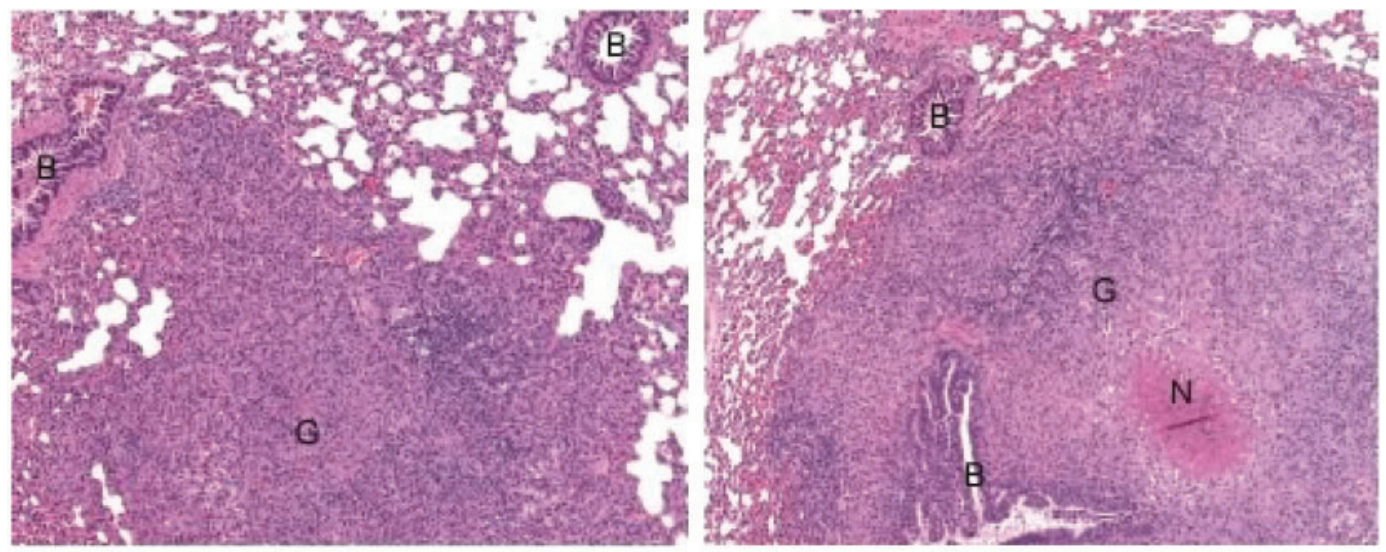

H37Rv

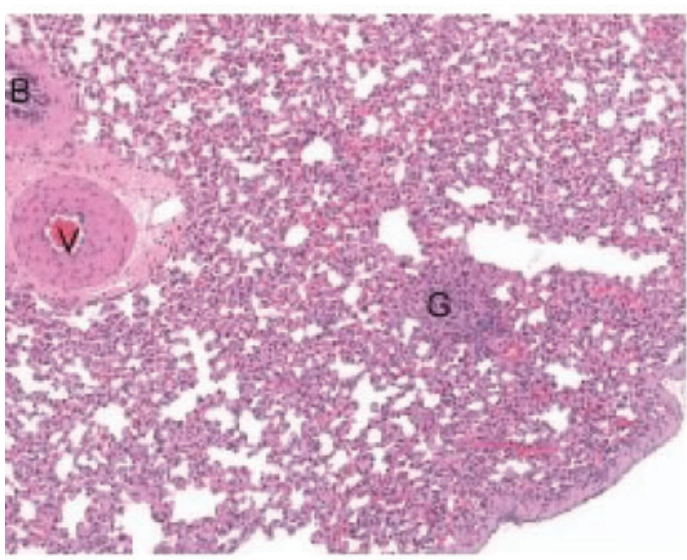

sigChyg sigMhyg

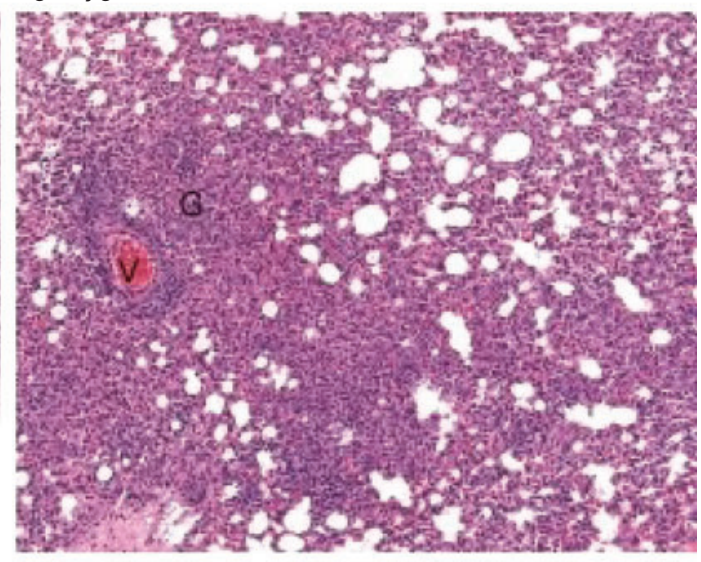

sigFhyg

Fig. 3. Photomicrographs of histopathological preparations of lungs infected with the different mutants at 5-6 weeks postinfection. Medium and extra large, well-formed granulomas $(G)$ are evident in guinea pigs infected with H37Rv and sigMhyg, respectively. The granuloma formed with sigMhyg also showed a central necrotic $(\mathrm{N})$ core. A small, well-formed granuloma was found with sigChyg. The sigFhyg strain resulted in ill-defined granulomas. Bronchioles (B) and prominent blood vessels (V) are labelled for reference. (Haematoxylin and eosin stains, original magnification $25 \times$.)

early and late times following infection with strains carrying a wild-type copy of sigC (H37Rv, sigMhyg, sigFhyg, sigCcomp), only a single small granuloma was detected in an animal 6 weeks post-infection with one of the sigC mutants (sigChyg). The granulomas from the sigFhyginfected animals appeared less-defined in the lung compared to the spleen at early and late timepoints (Figs 3, 4 and 5, Table 2). Granulomas with detectable AFB were observed in the spleen of animals at 5-6 weeks following infection with H37Rv, sigMhyg, sigFhyg, but not with either sigC knockout mutant or, interestingly, the complemented strain (sigCcomp, Table 2). At 20 weeks postinfection, granulomas with detectable AFB were observed in the spleen of animals infected with either H37Rv or sigFhyg, suggesting that these granulomas were induced by the presence of M. tuberculosis bacilli (Table 2). While a granuloma was detected in the spleen from one animal infected with $R v$-sigC at 20 weeks post-infection, no associated AFB were found. Therefore, we can not formally conclude that this granuloma was induced by mycobacteria.
Necrosis within granulomas was evident in the lungs of animals 5-6 weeks post-infection with H37Rv or sigMhyg, and in spleens infected with H37Rv, sigMhyg or sigFhyg (Table 2, Figs 3, 4 and 5). While a granuloma was detected in the lung of an animal infected with the complemented sigC mutant strain ( $\operatorname{sig} C \operatorname{comp})$, the absence of necrosis was unexpected. This may have been due to aberrant expression of a wild-type copy of sigC integrated at the L5 mycobacteriophage attachment site on the chromosome. Calcification of the necrotic centres was evident in lung granulomas of animals infected with either H37Rv or sigMhyg at 20 weeks post-infection (Table 2, Fig. 5). The lack of necrosis in granulomas from any animal infected with either sigC knockout (sigChyg or $R v$-sigC) suggests that SigC regulates factors important for host immune modulation.

\section{DISCUSSION}

In this study, M. tuberculosis mutants of sigC, sigF and sigM were created in strain $\mathrm{H} 37 \mathrm{Rv}$ and compared with one 


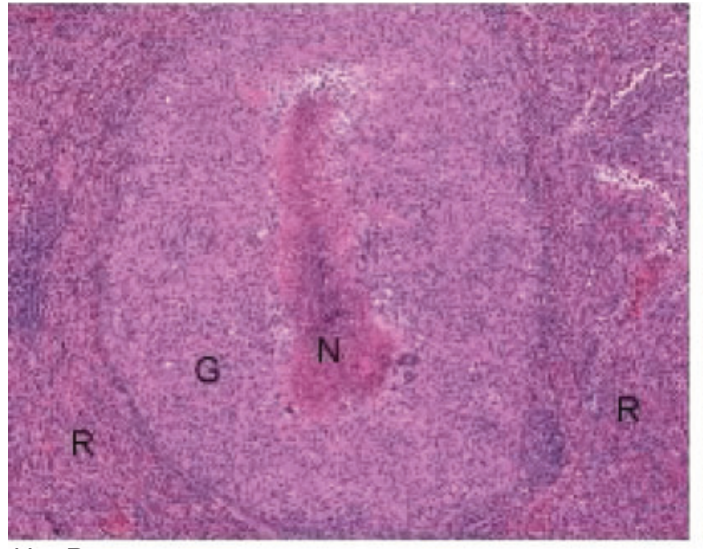

H37Rv

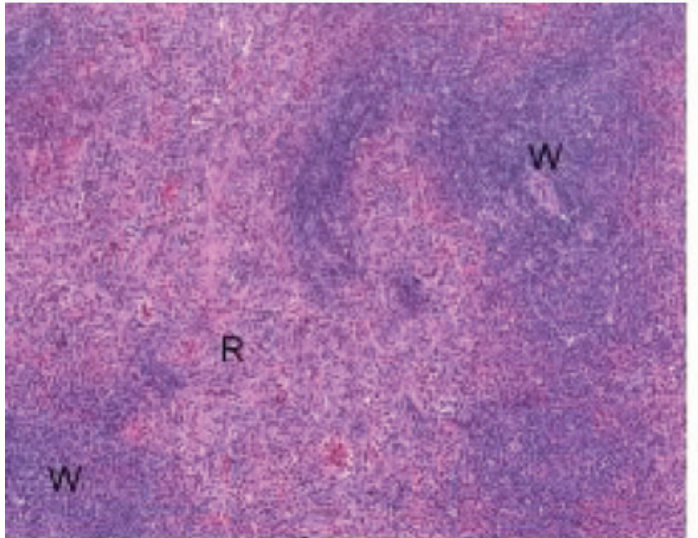

sigChyg

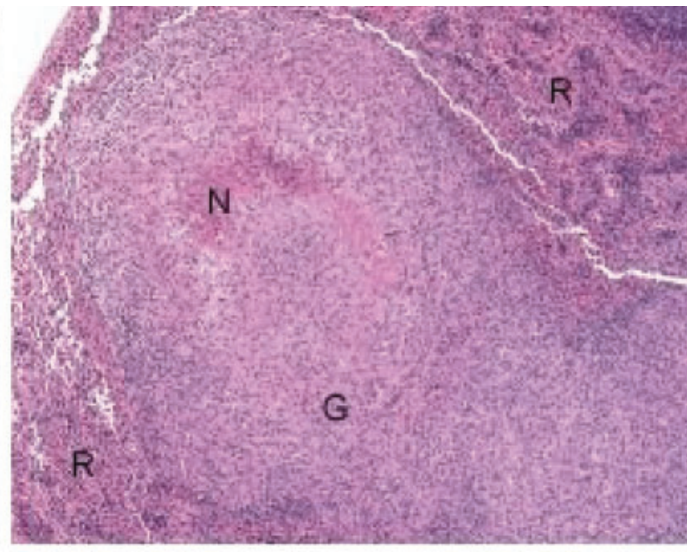

sigMhyg

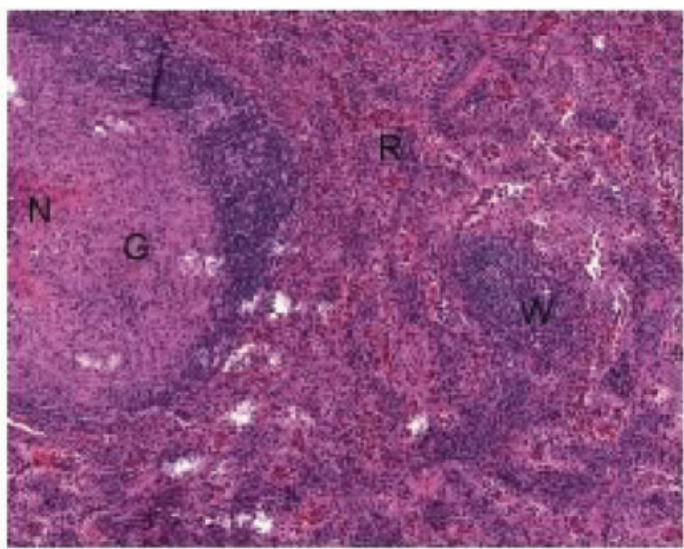

sigFhyg

Fig. 4. Photomicrographs of histopathological preparations of spleens infected with the different mutants at 5-6 weeks postinfection. Large, well-formed granulomas $(\mathrm{G})$ with necrotic $(\mathrm{N})$ core are noted for guinea pigs infected with H37Rv, sigFhyg, or sigMhyg. Spleen tissue from the sigChyg-infected animals appeared relatively normal, consisting of erythrocyte-enriched sinusoids [also known as red pulp (R)] and lymphocyte-enriched white pulp (W). (Haematoxylin and eosin stains, original magnification $25 \times$.)

another for alterations in growth and virulence phenotypes in vitro and in the guinea pig model of TB pathology. The lack of growth differences in broth cultures among the mutants and parent strain indicates that none of these sigma factors is critical for survival under non-stressed conditions. This is consistent with transposon site hybridization analyses that previously indicated that the genes encoding these sigma factors were non-essential for in vitro growth (Sassetti et al., 2003). The ability of all the mutants to replicate inside THP-1 macrophages suggests that none of these sigma factors exclusively regulate genes that are critical for survival in macrophages. The ability of the $\operatorname{sigC}$ and sigF mutants of strain $\mathrm{H} 37 \mathrm{Rv}$ to replicate in macrophages is consistent with reports of replication in macrophages by $s i g C$ and $s i g F$ mutants derived from $M$. tuberculosis strain CDC1551 (Sun et al., 2004; Chen et al., 2000).

The goal of this work was to use the guinea pig model as a screen for M. tuberculosis sigma factor mutants to identify those that mediate adaptive survival and pathogenesis upon entering the host environment. Although different murine strains have been used to model the relevance of specific sigma factors to human TB disease, this is believed to be the first reported use of the guinea pig model to examine the contributions of sigma factors to M. tuberculosis virulence. To mimic the typical route of human infection, the animals were infected via low-dose aerosols that deliver approximately 30 c.f.u. of bacilli to the lungs. Reflecting the outbred nature of guinea pigs, variability in tissue pathology was evident among replicate animals infected with the same bacterial strain (Table 2). However, general differences in granuloma size and composition were detectable among the sigma factor mutants. A sigM mutant was the least attenuated. It resulted in necrotic granulomas in the lungs and spleen much like those observed in H37Rvinfected animals. In addition, as with H37Rv-infected animals, calcification of lung granulomas was evident at 20 weeks post-infection. Thus, it would seem that SigM is not essential for virulence in this host. 

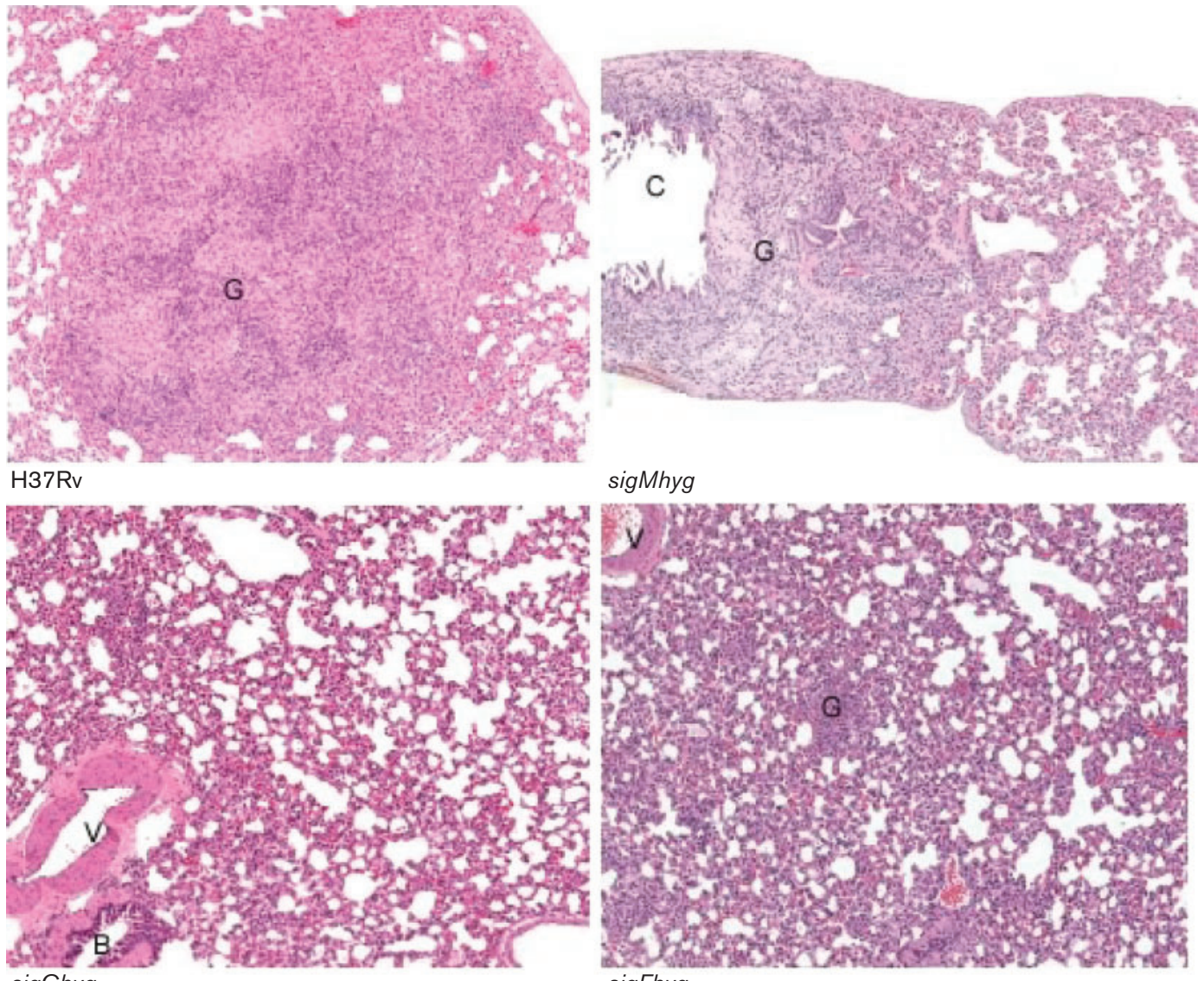

sigMhyg

sigChyg

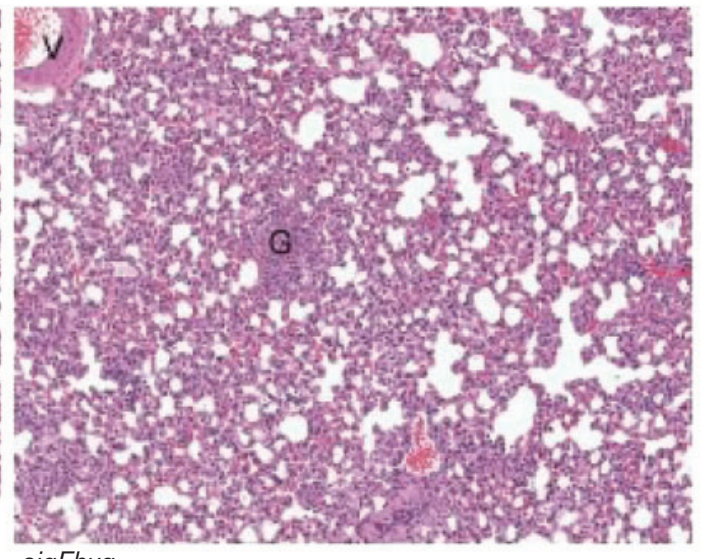

sigFhyg

Fig. 5. Photomicrographs of histopathological preparations of lungs infected with the different mutants at 20 weeks postinfection. Large, well-formed granulomas $(G)$ are noted for guinea pigs infected with H37Rv or sigMhyg. The granuloma formed with the sigMhyg mutant also showed a central necrotic and calcified (C) core. The guinea pig infected with the sigFhyg mutant contained a small, non-necrotic granuloma (G). Relatively normal 'lacelike' appearance of healthy lung tissue due to the absence of alveolar airsac staining is observed from animals infected with the sigChyg mutant. Bronchi (B) and blood vessels $(\mathrm{V})$ are also indicated. (Haematoxylin and eosin stains, original magnification $25 \times$.)

A sigF mutant resulted in diffuse guinea pig lung granulomas lacking necrosis, but surprisingly normal-appearing granulomas with necrotic centres in the spleen. The lung phenotype is generally consistent with a murine study of a sigF mutant of CDC1551 which reported reduced lung granulomatous inflammation relative to the parent strain between 12 and 20 weeks post-infection (Geiman et al., 2004). The presence of necrotic guinea pig spleen granulomas containing AFB suggests that differences in the internal environments of these organs affect the survival of SigF-deficient bacilli. Geiman et al. (2004) reported a reduction in envelope-associated sulfolipids in a sigF mutant. Reduced amounts of this or other SigF-regulated products may result in heightened susceptibility to oxidizing agents or surfactants found exclusively in the lungs of guinea pigs and mice.

Studies of the three M. tuberculosis sigma factor mutants in guinea pigs suggest that $\mathrm{SigC}$ is more important for pathogenesis and survival within granulomas than either SigF or SigM. SigC-deficient bacilli resulted in fewer and smaller lung and spleen granulomas. In addition, necrotic centres were absent from granulomas in the lung of animals infected with a $\operatorname{sig} C$ mutant. This suggests that SigC is an important regulator of virulence. This view is consistent with observations that a sigC mutant of a recent clinical M. tuberculosis strain (CDC1551) produced fewer granulomas in DBA2 mice (Sun et al., 2004). However, a difference in persistence of $\operatorname{sig} C$ mutants between these models may exist. In this study, the lack of lung granulomas or detectable AFB suggests that sigC mutants derived from strain $\mathrm{H} 37 \mathrm{Rv}$ do not persist to 20 weeks postinfection. This contrasts with the report that CDC1551 sigC mutant bacilli maintained elevated lung titres for a comparable period (Sun et al., 2004). This difference in persistence may be due to inherent differences between H37Rv and CDC1551. Alternatively, physiological differences between these animal models might also explain 
Table 2. Histopathological analysis of guinea pig organs following infection with $M$. tuberculosis sigma factor mutants

Pathological assessments were made blinded to the identities of the infecting strains (left column). Equivalent amounts of tissue were examined from each animal.

\begin{tabular}{|c|c|c|c|c|c|c|c|c|c|c|}
\hline \multirow[t]{2}{*}{ Strain } & \multirow{2}{*}{$\begin{array}{c}\text { Time } \\
\text { (weeks) }\end{array}$} & \multirow{2}{*}{$\begin{array}{c}\text { No. of } \\
\text { pigs tested }\end{array}$} & \multicolumn{4}{|c|}{ Lungs } & \multicolumn{4}{|c|}{ Spleen } \\
\hline & & & $\begin{array}{l}\text { No. of pigs with } \\
\text { granulomas }\end{array}$ & $\begin{array}{l}\text { Granulomas } \\
\text { and size }\end{array}$ & $\begin{array}{l}\text { Granulomas } \\
\text { with necrosis }\end{array}$ & $\begin{array}{l}\text { Granulomas with } \\
\text { AFB (no. of AFB) }\end{array}$ & $\begin{array}{l}\text { No. of pigs with } \\
\text { granulomas }\end{array}$ & $\begin{array}{c}\text { Granulomas } \\
\text { and size } \dagger\end{array}$ & $\begin{array}{l}\text { Granulomas } \\
\text { with necrosis }\end{array}$ & $\begin{array}{l}\text { Granulomas with } \\
\text { AFB (no. of AFB) }\end{array}$ \\
\hline $\mathrm{H} 37 \mathrm{Rv}$ & 6 & 4 & 4 & $4 \mathrm{MED}, 4 \mathrm{SM}$ & 1 & $1(2)$ & 2 & $>3 \mathrm{LG}, 2 \mathrm{MED}$ & 1 & $2(3-4)$ \\
\hline sigMhyg & 6 & 4 & 4 & $>3 \mathrm{LG},>7 \mathrm{MED}$ & 2 & $2(4-10)$ & 1 & $>3 \mathrm{LG}$ & 1 & $1(4-6)$ \\
\hline sigFhyg & 6 & 4 & 3 & $2 \mathrm{MED} \ddagger, 1 \mathrm{SM} \ddagger$ & 0 & 0 & 2 & $2 \mathrm{LG}, 1 \mathrm{MED}$ & 1 & $2(1-2)$ \\
\hline sigChyg & 6 & 4 & 1 & $1 \mathrm{SM}$ & 0 & 0 & 0 & 0 & 0 & 0 \\
\hline $\mathrm{H} 37 \mathrm{Rv}$ & 17 & 4 & 3 & $>3 \mathrm{LG}, 3 \mathrm{SM}$ & 1 & 0 & 0 & 0 & 0 & 0 \\
\hline sigMhyg & 17 & 4 & 2 & $>6 \mathrm{XLG}$ & 1 & $3(4-10)$ & 3 & $>3 \mathrm{XLG},>3 \mathrm{MED}$ & 1 & $1(>10)$ \\
\hline sigFhyg & 17 & 4 & 0 & 0 & 0 & 0 & 1 & 5 MED & 0 & $1(1)$ \\
\hline sigChyg & 17 & 4 & 0 & 0 & 0 & 0 & 1 & $2 \mathrm{SM}$ & 0 & 0 \\
\hline $\mathrm{H} 37 \mathrm{Rv}$ & 20 & 2 & 2 & $4 \mathrm{LG}$ & 2 (calcified) & 0 & 1 & $3 \mathrm{LG}$ & 1 & $1(4-10)$ \\
\hline sigMhyg & 20 & 2 & 1 & $1 \mathrm{LG}$ & 1 (calcified) & 0 & 0 & 0 & 0 & 0 \\
\hline sigFhyg & 20 & 2 & 1 & $1 \mathrm{SM}$ & 0 & 0 & 1 & $1 \mathrm{XLG}$ & 1 & $1(4-10)$ \\
\hline sigChyg & 20 & 2 & 0 & 0 & 0 & 0 & 0 & 0 & 0 & 0 \\
\hline$R v$-sigC & 5 & 4 & 0 & 0 & 0 & 0 & 0 & 0 & 0 & 0 \\
\hline sigCcomp & 5 & 4 & 2 & $3 \mathrm{MED}, 1 \mathrm{SM}$ & 1 & 0 & 0 & 0 & 0 & 0 \\
\hline$R v$-sigC & 20 & 4 & 0 & 0 & 0 & 0 & 1 & $1 \mathrm{SM}$ & 0 & 0 \\
\hline sigCcomp & 20 & 4 & 2 & $>5 \mathrm{MED}, 2 \mathrm{SM}$ & 0 & 0 & 0 & 0 & 0 & 0 \\
\hline
\end{tabular}

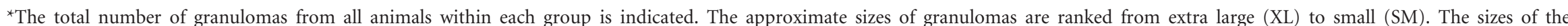

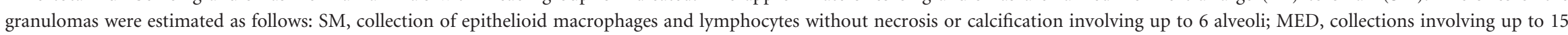
alveoli with or without necrosis; LG, coalescent collections with central necrosis but not larger than 30 alveoli; XLG, coalescent collections with central necrosis larger than 30 alveoli.

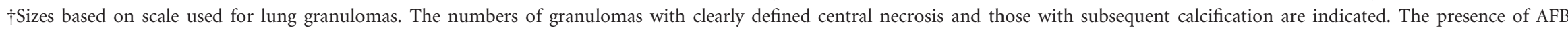
within granulomas is also indicated.

‡Denotes ill-defined granulomas. 
the divergent survival. The ability of guinea pigs to produce granulomas with necrotic cores may result in effective killing of internalized $M$. tuberculosis compared to murine hosts, thereby minimizing dissemination.

The differences in guinea pig lung and spleen granulomas induced by $\operatorname{sig} C$ or sigF mutants suggest different mechanisms of resisting host defences. A sigF mutant maintains the ability to produce necrotic granulomas in the spleen, but produces ill-defined lung granulomas. In contrast, sigC mutants fail to cause the production of necrotic granulomas in either organ. Different promoter consensuses have been proposed for SigC and SigF, suggesting that each controls a unique regulon (Sun et al., 2004; Geiman et al., 2004). Given that each mutant produces limited lung pathology, it is possible that some genes may have promoters for both sigma factors. Expression of sigC was reported to be downregulated in a sigF mutant in early stationary phase growth (Geiman et al., 2004). The granuloma phenotypes are consistent with such a hierarchical regulation and suggest that $\mathrm{SigC}$ is more likely than SigF to be a direct regulator of genes that enable the bacilli to successfully adapt to critical host defences. Future efforts will seek to define the factors directly regulated by SigC, as they may serve as targets for vaccines or anti-mycobacterial therapies.

These studies support the hypothesis that SigC, and to a lesser extent SigF, is responsible for mediating adaptive survival of $M$. tuberculosis upon entering the host environment. It remains unclear what host signals activate expression or activity of these sigma factors. Further studies examining virulence of strains grown to stationary phase or taken from the surface pellicle of cells cultured under nonshaking conditions may result in bacilli expressing various stress response factors prior to entering the host. Bacilli pre-armed as such prior to infection would be expected to be more resistant upon initial encounter with host cells and may be more adept at redirecting the immune response away from a cell-mediated killing of this intracellular pathogen.

\section{ACKNOWLEDGEMENTS}

This research was supported by an appointment (R.K.K.) to the Research Participation Program at the CDC, National Center for Infectious Diseases, Division of AIDS, STD, and TB Laboratory Research and administered by the Oak Ridge Institute for Science and Education through an interagency agreement between the US Department of Energy and CDC. R. K. K. was also funded through the ASM/NCID Postdoctoral Research Associateship program.

We thank Pei-Hsiu Huang, Jason Ho, Nicole Latorre, Christine Gilmore and Sara Smiley for assistance in construction of vectors used in creating sigma factor mutants. We thank Lauren Jodi Pitkow for performing Southern blots confirming PCR analyses of the mutants. We are grateful to Susan Phalen and Christine McFarland for their assistance with the animal infections and necropsies. We thank Jeanine Bartlett for preparation of histopathological material. We thank Thomas Shinick and Jack Crawford for helpful discussions and critical reading of this paper.

\section{REFERENCES}

Ando, M., Yoshimatsu, T., Ko, C., Converse, P. J. \& Bishai, W. R. (2003). Deletion of M. tuberculosis sigma factor E results in delayed time to death with bacterial persistence in the lungs of aerosolinfected mice. Infect Immun 71, 7170-7172.

Baldwin, S. L., D'Souza, C., Roberts, A. D. \& 7 other authors (1998). Evaluation of new vaccines in the mouse and guinea pig model of TB. Infect Immun 66, 2951-2959.

Chen, P., Ruiz, R. E., Li, Q., Silver, R. F. \& Bishai, W. R. (2000). Construction and characterization of a $M$. tuberculosis mutant lacking the alternate sigma factor gene, sigF. Infect Immun 68, 5575-5580.

Cole, S. T., Brosch, R., Parkhill, J. \& 38 other authors (1998). Deciphering the biology of $M$. tuberculosis from the complete genome sequence. Nature 393, 537-544.

Cotran, R. S., Kumar, V. \& Collins, T. (1999). Robbins Pathologic Basis of Disease, 6th edn. Philadelphia, PA: W. B. Saunders.

Dye, C., Scheele, S., Dolin, P., Pathania, V. \& Raviglione, M. C. (1999). Consensus statement. Global burden of tuberculosis: estimated incidence, prevalence, and mortality by country. WHO Global Surveillance and Monitoring Project. JAMA 282, 677-686.

Erickson, J. W. \& Gross, C. A. (1989). Identification of the $\sigma^{\mathrm{E}}$ subunit of Escherichia coli RNA polymerase: a second alternate $\sigma$ factor involved in high-temperature gene expression. Genes Dev 1, 419-432.

Geiman, D. E., Kaushal, D., Ko, C. \& 8 other authors (2004). Attenuation of late-stage disease in mice infected by the $M$. tuberculosis mutant lacking the SigF alternate sigma factor and identification of SigF-dependent genes by microarray analysis. Infect Immun 72, 1733-1745.

Gonzalez-Juarrero, M., Turner, O. C., Turner, J., Marietta, P., Brooks, J. V. \& Orme, I. M. (2001). Temporal and spatial arrangement of lymphocytes within lung granulomas induced by aerosol infection with M. tuberculosis. Infect Immun 69, 1722-1728.

Hu, Y., Kendall, S., Stoker, N. G. \& Coates, A. R. (2004). The M. tuberculosis sigJ gene controls sensitivity of the bacterium to hydrogen peroxide. FEMS Microbiol Lett 237, 415-423.

Kaushal, D., Schroeder, B. G., Tyagi, S. \& 8 other authors (2002). Reduced immunopathology and mortality despite tissue persistence in a $M$. tuberculosis mutant lacking alternative sigma factor, SigH. Proc Natl Acad Sci U S A 99, 8330-8335.

Kong, D. \& Kunimoto, D. Y. (1995). Secretion of human interleukin 2 by recombinant $M$. bovis BCG. Infect Immun 63, 799-803.

Manganelli, R., Voskuil, M. I., Schoolnik, G. K. \& Smith, I. (2001). The M. tuberculosis ECF sigma factor $\sigma^{\mathrm{E}}$ : role in global gene expression and survival in macrophages. Mol Microbiol 41, 423-437.

Manganelli, R., Fattorini, L., Tan, D., Iona, E., Orefici, G., Altavilla, G., Cusatelli, P. \& Smith, I. (2004). The extra cytoplasmic function sigma factor $\sigma^{\mathrm{E}}$ is essential for M. tuberculosis virulence in mice. Infect Immun 72, 3038-3041.

McMurray, D. N., Collins, F. M., Dannenberg, A. M., Jr \& Smith, D. W. (1996). Pathogenesis of experimental TB in animal models. Curr Top Microbiol Immunol 215, 157-179.

Orme, I. M., McMurray, D. N. \& Belisle, J. T. (2001). Tuberculosis vaccine development: recent progress. Trends Microbiol 9, 115-118.

Parish, T. \& Stoker, N. G. (2000). Use of a flexible cassette method to generate a double unmarked $M$. tuberculosis tlyA plcABC mutant by gene replacement. Microbiology 146, 1969-1975.

Pelicic, V., Jackson, M., Reyrat, J. M., Jacobs, W. R., Jr, Gicquel, B. \& Guilhot, C. (1997). Efficient allelic exchange and transposon 
mutagenesis in M. tuberculosis. Proc Natl Acad Sci U S A 94, 10955-10960.

Raman, S., Hazra, R., Dascher, C. C. \& Husson, R. N. (2004). Transcription regulation by the $M$. tuberculosis alternative sigma factor SigD and its role in virulence. J Bacteriol 186, 6605-6616.

Rhoades, E. R., Frank, A. A. \& Orme, I. M. (1997). Progression of chronic pulmonary tuberculosis in mice aerogenically infected with virulent M. tuberculosis. Tubercle Lung Dis 78, 57-65.

Ridley, D. S. \& Ridley, M. J. (1987). Rationale for the histological spectrum of TB. A basis for classification. Pathology 19, 186-192.

Rodrigues, L. \& Smith, P. G. (1990). Tuberculosis in developing countries and methods for its control. Trans $R$ Soc Trop Med Hyg 84, 739-744.

Sassetti, C. M., Boyd, D. H. \& Rubin, E. J. (2003). Genes required for mycobacterial growth defined by high density mutagenesis. Mol Microbiol 48, 77-84.

Sun, R., Converse, P. J., Ko, C., Tyagi, S., Morrison, N. E. \& Bishai, W. R. (2004). M. tuberculosis ECF sigma factor SigC is required for lethality in mice and for the conditional expression of a defined gene set. Mol Microbiol 52, 25-38.

Turner, O. C., Basaraba, R. J. \& Orme, I. M. (2003). Immunopathology of pulmonary granulomas in the guinea pig after infection with M. tuberculosis. Infect Immun 71, 864-871.

Wiegeshaus, E. H., McMurray, D. N., Grover, A. A., Harding, G. E. \& Smith, D. W. (1970). Host-parasite relationships in experimental airborne TB. III. Relevance of microbial enumeration to acquired resistance in guinea pigs. Am Rev Respir Dis 102, 422-429.

World Health Organization (1979). Trial of BCG vaccines in South India for TB prevention. WHO Bull 57, 810-827.

World Health Organization (2005). Fact sheet no. 104 (revised April 2005). Tuberculosis. http://www.who.int/mediacentre/factsheets/fs104/ en/print.html.

Yuan, Y., Crane, D. D., Simpson, R. M., Zhu, Y. Q., Hickey, M. J., Sherman, D. R. \& Barry, C. E., 3rd (1998). The 16-kDa alphacrystallin (Acr) protein of M. tuberculosis is required for growth in macrophages. Proc Natl Acad Sci U S A 95, 9578-9583. 